\title{
Representaciones Sociales sobre la Salud y Enfermedad Oral en familias de La Boquilla Cartagena-Colombia
}

\author{
Farith González Martínez ${ }^{a}$, Edwin Puello del Rio ${ }^{b}$, Antonio Díaz Caballeroc
}

a Odontólogo, Magíster en Salud Pública, Departamento de Investigación Facultad de Odontología, Universidad de Cartagena - Colombia.

b Odontólogo, Gerente en Salud, Coordinador Académico Universidad de Cartagena, Colombia.

c Odontólogo, Magíster en Educación, Departamento de Medicina Oral, Facultad de Odontología, Universidad de Cartagena, Colombia.

Correspondencia:

Farith González Martínez,

Facultad de Odontología, Universidad de Cartagena, Campus de la Salud,

Departamento de

Investigación, Barrio

Zaragocilla, Cartagena de

Indias, Colombia.

Telf.: 095-6698184, ext. 110, e-mail: farithgm@hotmail.com antoniodiazc@yahoo.com.

Recibido el 26 de octubre de 2009.

Aceptado para su publicación el 19 de diciembre de 2009.

\section{RESUMEN}

Objetivo. Describir las representaciones sociales que orientan la construcción de conocimientos sobre salud y enfermedad oral en las familias del municipio de La Boquilla, Cartagena.

Diseño. Estudio cualitativo, a través de una aproximación al método etnográfico.

Emplazamiento. Municipio de La Boquilla Cartagena - Colombia.

Participantes. 15 familias del sector, con el fin de obtener la información útil para los objetivos del estudio.

Mediciones principales. Representaciones sobre la etiología, prevención y tratamientos de la enfermedad bucal, origen y significado de los conocimientos en salud oral.

Resultados. Se evidenciaron interpretaciones sobre la salud y la enfermedad oral, que estaban influenciadas por la cultura, el trabajo, la pobreza y las emociones, elementos éstos poco racionales desde lo científico, los cuales logran reconocer a un ser social que puede utilizar comportamientos y actitudes saludables con explicaciones diferentes a la cultura occidental, pero tratando de buscarle una solución al problema desde diferentes dimensiones.

Conclusiones. Las representaciones sociales de la población de La Boquilla - Cartagena ejercen una influencia en la apropiación de un conocimiento útil y aplicable dentro de su núcleo familiar, hasta el punto de lograr confrontarlo con el conocimiento científico que transmiten los profesionales de la salud, y de esta forma sacar conclusiones individuales basadas en una reflexión desde una perspectiva empírica.

Palabras clave. Interacción Social, Promoción de la Salud, Prevención Primaria, Etnografía.

\section{ABSTRACT}

Social Representations on the Oral Health and Disease in the families living in La Boquilla Cartagena-Colombia

Objective. To describe the representations that guide the social construction of knowledge on oral health and disease in families living in the town of La Boquilla Cartagena.

Design. Qualitative study, using an ethnographical method.

Setting. The town of La Boquilla Cartagena - Colombia.

Participants. 15 families from the twon in order to obtain useful information for the purpose of the study.

Main measurements. Representations on the aetiology, prevention and treatment of oral disease, origin and meaning of oral health knowledge.

Results. We found interpretations on health and oral disease, which were influenced by culture, work, poverty and emotions, scientifically unsound, which are able to recognize a social being with certain health attitudes and behaviour different from those of Western culture, but trying to find a solution to the problem from different dimensions.

Conclusions. The social representations on health and oral disease in the town of La Boquilla - Cartagena, exert an influence on the appropriation of useful knowledge applicable to the family, to the point of challenging scientific knowledge transmitted by health professionals and thus draw conclusions based on an individual reflection from an empirical perspective.

Key words. Interpersonal Relations, Health Promotion, Primary Prevention, Anthropology, Culture. 


\section{INTRODUCCIÓN}

Varios estudios han descrito a nivel mundial la asociación entre las condiciones sociales y la percepción de estados de la salud oral. En Colombia en 2004 realizamos un estudio epidemiológico en cavidad oral ${ }^{1}$, evaluando los conocimientos sobre higiene oral en padres y niños escolarizados de esta población. Los resultados obtenidos fueron muy superiores a los del Estudio Nacional de Salud Bucal ${ }^{2}$ (ENSAB III 1998), en lo referente a caries dental, (COP-D 13.0 y COP-S 24.3) enfermedad periodontal, sangrado (46\%), cálculos (39\%) y fluorosis $(41.9 \%)$. En cuanto a los conocimientos, se observó un alto porcentaje de respuestas adecuadas en todos los grupos, observando mejores resultados en niños entre 12 y 14 años. Sin embargo, aún no se pudo aclarar cómo se construye el conocimiento sobre la salud oral al interior de la comunidad, cuáles son sus significados y qué influencia pueden estar ejerciendo sus representaciones sociales.

A nivel nacional se han evidenciado algunas aproximaciones, entre las necesidades de las comunidades y el trabajo de los profesionales de la odontología, reconociendo que las nuevas tendencias en salud oral están encaminadas hacia la prevención de las enfermedades desde un componente educativo $^{3,4}$. No obstante, a nivel del Sistema General de Seguridad Social en Salud ${ }^{5}$ (SGSSS) se han encontrado deficiencias en torno al manejo pedagógico que las instituciones de salud promueven en la población, ya que se diseñan programas de promoción de la salud y prevención de la enfermedad, con el único propósito de aumentar coberturas, incluyendo conocimientos en forma generalizada, tratando de esta forma de homogeneizar a la población, sin tener en cuenta la educación que han recibido, sus condiciones económicas y culturales, la facilidad de acceso al servicio y, sobre todo, las diferentes conceptualizaciones e imaginarios que éstos han construido sobre la salud y enfermedad oral.

Todos estos factores pueden ser las causales de que las acciones educativas y preventivas no sean apropiadas por los habitantes de estas comunidades, lo cual se refleja en la baja efectividad de las intervenciones de los servicios de salud oral ${ }^{6}$.

El presente estudio propone develar este paradigma, describiendo las representaciones sociales que orientan la construcción de conocimientos sobre salud y enfermedad oral en las familias de una localidad rural del caribe colombiano, con el fin de incorporarlas en las prácticas de higiene oral que promueven los profesionales de la odontología.

\section{MATERIAL Y MÉTODO}

Se planteó un estudio cualitativo, realizado en el municipio de La Boquilla Cartagena - Colombia, entre los años 2007 y 2008, utilizando una aproximación al método etnográfico. Los participantes fueron 15 familias del sector, seleccionadas teniendo en cuenta la probabilidad de obtener información útil para los objetivos del estudio, lo que necesitó de un acercamiento a los procesos de entendimiento y comunicación de los sujetos dentro de los grupos sociales ${ }^{7,8}$.

Como instrumentos de recogida de la información se realizaron: a) entrevistas individuales estructuradas a 15 madres y/o padres cabezas de familia de la población; b) entrevistas en profundidad a tres lideres comunitarios, los cuales por tradición son reconocidos en la población como portadores de un saber popular; c) guión para grupos focales, aplicado a ocho madres de familia con el fin de profundizar en la información que todavía no había llegado a un estado de saturación, tratando de ajustar o construir categorías emergentes; d) entrevistas individuales no estructuradas a siete tenderos de la zona evaluada; e) entrevistas individuales semiestructuradas a dos profesionales que trabajan en el puesto de salud de la población y a tres usuarios del servicio al momento de acceder a la consulta; f) diarios de campo y observación participante durante las visitas domiciliarias a 15 hogares de la zona, las cuales tenían niños en los diferentes colegios del corregimiento; g) observación no participante durante la fase exploratoria del estudio, para obtener un acercamiento a las interacciones sociales de la población, utilizando actividades lúdicas con ocho niños escolarizados, para acercarnos a los simbolismos con que estos reconocen la salud y la enfermedad oral.

El trabajo de campo se inició con la caracterización de la población objeto de estudio respecto a los aspectos geográfico, histórico, sociocultural, familiar, económico y en salud oral, con el fin de adquirir las competencias hermenéuticas necesarias para la recogida de los datos. Posteriormente se seleccionaron las familias, diligenciando un consentimiento informado por escrito para los padres responsables de los niños, explicándoles que este estudio no generaba ningún riesgo para la salud de los participantes y que los investigadores adquirían el compromiso de no vulnerar su intimidad con la publicación de la información recolectada, todo esto teniendo en cuenta lo dispuesto en la resolución 84301993 del Ministerio de Salud República de Colombia y en la declaración de Helsinki, modificación de Edimburgo año 2000. Luego se aplicaron las técnicas 
e instrumentos para la recogida de la información, teniendo en cuenta las categorías: etiología, prevención y tratamiento de la enfermedad bucal, origen y significado de los conocimientos en salud oral, procesando y analizando los datos obtenidos. Para este fin, durante todo el proceso y desde el momento de la categorización inicial se realizó una lectura permanente de la información, agrupándola en categorías previamente planteadas y en las que emergieron del análisis. Además, de una relectura de la información, estableciendo subcategorías y aproximándonos a la interpretación, teniendo como guías los referentes teóricos.

Luego de definidas las categorías y subcategorías, se realizaron cruces de la información obtenida en las diferentes fuentes, surgiendo comentarios triangulados, buscando patrones temáticos, relaciones y concurrencias entre cada párrafo e identificación de metáforas y concordancias en las diferentes respuestas.

\section{RESULTADOS}

\section{Representaciones sociales sobre salud y enfer- medad}

La salud y la enfermedad oral desde lo biológico: en este tópico los individuos dan explicaciones a las enfermedades refiriéndose a signos y síntomas, reconociéndolas como eventos importantes cuando se consulta al odontólogo. De esta forma, se asumen conceptualizaciones deterministas, en las cuales se encuentra explicada la salud con la posibilidad de tener un buen aseo o higiene oral. Dentro de las descripciones de salud que más se presentan están: "Una buena salud oral es que la persona goce de una buena dentadura, eh que no tenga muelas picadas, no tenga caries, esa es una buena salud para mí". Aquí se asocia la buena dentadura con la salud oral. De igual forma se relaciona la salud con la sensación de limpieza en la boca, representada por los buenos olores, lo cual refleja un entendimiento con mayor énfasis hacia el aseo que a la función.

La salud y la enfermedad oral desde lo estético: aquí se relaciona la salud oral con lo limpio, blanco y agradable a los sentidos. Con estos elementos los individuos tratan de explicar una connotación estética influida por varios aspectos: la intervención de las imágenes estereotipadas construidas desde la interpretación del mensaje de algunos medios de comunicación y el efecto publicitario del mercantilismo comercial, en la cuál se trata de colocar a la salud oral dentro de la perfección, ligada a una imagen visual que propende por cambiar la concepción estética de los individuos con estilos de vida diferentes, hacia formas "homogéneas" de percibir la belleza. En este sentido, las opiniones giran en torno a la concepción de una buena higiene como mecanismo para cuidar los dientes, simplemente pensando en verlos limpios y blancos, sin importar su naturaleza, su función masticatoria y las demás funciones del sistema estomatognático. En este orden de ideas se encuentran expresiones de salud como "Tener los dientes sanos es no tener mal aliento ni desperfectos en su boca, utilizando la higiene". Además de darle importancia al buen aliento, se vincula la parte estética, reconociendo la enfermedad como un elemento de imperfección, lo que determina un condicionamiento de la salud hacia la belleza.

\section{Representaciones sobre la etiología de la enfer- medad oral}

Consideraciones desde lo exógeno y endógeno: en este sentido se tienen en cuenta todos los elementos que explican a la enfermedad analizándose desde sus signos y síntomas e incluyendo factores asociados como la alimentación y la higiene oral. Esto se pudo apreciar al preguntarles el por qué se enferman los dientes, encontrando expresiones en estas dos vías: "Sí, porque hay muchas personas que no utilizan el correcto cepillado y por los alimentos, por los dulces, muchos niños y adultos comen dulces, y al acostarse no se cepillan". Esta explicación se fundamenta en elementos multifactoriales (alimentación y hábitos higiénicos orales). De esta forma se le está dando un origen a la enfermedad oral desde el punto de vista externo y se reconstruye la creencia de que el metabolismo de los alimentos le aporta nutrientes a los dientes a cualquier edad, lo que contribuye a fortalecerlos 0 , por el contrario, a debilitarlos por el pobre consumo de proteínas y minerales.

Desde la observación se pudo establecer que el estilo de vida de los pobladores, especialmente entre los hombres, puede estar influyendo en sus hábitos de higiene oral, debido a que en la mayoría de los casos se dedican a la pesca artesanal, iniciando sus actividades a partir de las tres de la mañana para ir en busca del sustento diario y pasan todo el día fuera del hogar, en el mar, y regresan por la tarde para vender su producto, llegando a casa por la noche con muy poca motivación para el aseo oral y teniendo como única expectativa el descanso y poder recuperar fuerzas para una nueva jornada. Este hecho ocasiona que pasen días sin cepillarse, estando expuestos a múltiples enfermedades orales, incluyendo las perdidas prematuras de órganos dentarios, con el correspondiente deterioro de su autoestima.

Cuando se indaga por el sangrado de las encías, se encuentran respuestas como "A veces es por el cepillo que es muy viejito y eso le parte, le puya la 
encía y eso le sangra". Otras de las respuestas que se encontraron para darle explicación al sangrado de las encías tiene que ver con el embarazo, donde se percibe un aspecto adicional: en primera instancia se resalta la inflamación de las encías como causa del daño de los dientes, y por otro lado se manifiesta la condición sistémica en el embarazo como una predisposición endógena para que se presenten estas enfermedades.

\section{Representaciones sobre la prevención de la en- fermedad oral}

Herramientas preventivas: las herramientas de mayor conocimiento en la población son el cepillo dental, la crema dental, en algunos casos el enjuague bucal y en muy pocas ocasiones el hilo dental. Se refleja entonces la influencia del discurso de los medios de comunicación para fortalecer las medidas preventivas. Además, el cepillo dental es valorado como de las más importantes estrategias. No obstante, cuando se pregunta en dónde se obtuvo este conocimiento, se observa un aprendizaje colectivo transmitido en una forma general sin ninguna explicación, resaltándose en la mayoría de los individuos sólo la importancia del odontólogo al recomendar el uso de estos elementos de higiene oral.

Teniendo en cuenta este entendimiento y a través de la observación y algunas preguntas no estructuradas que se le hicieron a tenderos del sector, se verificó que para la higiene oral sólo se encuentran cremas dentales para adultos de una marca comercial y cepillos dentales para adultos, sólo en algunos casos para niños. De igual forma, en ninguno de los sitios consultados se encontró la venta de seda dental, enjuague bucal u otros elementos de higiene oral, y los tenderos afirman que la demanda de estos productos no es muy continua. Con respecto a los precios, estos varían para las cremas dentales entre 1.500 y 4.000 pesos colombianos (US 0,75 y 2,0 respectivamente), dependiendo del tamaño, y para los cepillos dentales entre 1.000 y 1.500 pesos colombianos (US 0,50 y 0,75 respectivamente).

Por otro lado, con respecto al seguimiento del cepillado dental en los niños y la importancia que tiene evitar que ingieran la crema dental, se encuentra un desconocimiento total, resaltado en expresiones como "Le hace daño, le da mal de estomago", "Se traga el sucio de los dientes, pero es difícil evitarlo". En general, la problemática observada en la población con respecto a la prevención tiene que ver con que las personas saben cómo se previene, pero utilizan muy pocas herramientas preventivas, posiblemente porque no interiorizan este conocimiento para utilizarlo o no se conciencian de los beneficios que obtendrían dentro de la salud de la cavidad oral.

\section{Representaciones sobre los tratamientos de las enfermedades orales}

Dependiendo de la severidad de los síntomas, se pueden utilizar remedios caseros o comerciales para evitar que el cuadro se agrave. En otros casos se resalta la prioridad del remedio casero de forma inmediata cuando aparece el fenómeno y después de calmarlo se lleva al odontólogo para que el problema no se acentúe. Este conocimiento también se considera heredado desde el núcleo familiar y cuenta con un acervo popular, el cual, a través de la verificación empírica, logra resolver las inquietudes que se manifiestan por la presencia de las enfermedades.

Para las muelas huecas se utilizan como práctica los analgésicos dentro de la cavidad especialmente el ácido acetilsalicílico, el cual causa un efecto directo sobre el dolor de manera instantánea, pero los individuos no explican por qué funciona. Además, utilizan para quitar un dolor de muela la "Ramita de Jazmín" (Jasminum officinale L), "Azahar de la India" (Murraya paniculata), "Diente de León" (Taraxacum officinale L), una "Adormidera" (Papaver somniferum $L$ ) o en casos extremos se aplican un desinfectante fenólico concentrado en la cavidad dental con un algodón. Todos estos elementos tienen como indicación producir anestesia, pero en algunos casos el dolor desaparece por muerte pulpar, lo que puede agravar el problema a medio plazo.

Para las lesiones en la boca se utiliza Nistatina, medicamento indicado para las infecciones micóticas orofaríngeas. De igual forma, para las enfermedades de las encías, en especial la Periodontitis, cuando se observa movilidad por perdida de los tejidos de soporte del diente el único tratamiento es la exodoncia, la cual es la principal causa de dientes extraídos en los adultos de la población y uno de los principales motivos de consulta cuando asisten al puesto de salud. En caso de sangrado de las encías "Gingivitis" el tratamiento más común son los "Enjuagues de agua tibia", sin embargo, esto no se considera incapacitante, ni se le da mucha importancia dentro de las prioridades de los individuos.

En cuanto a los tratamientos realizados en el puesto de salud, las familias observadas piensan que solamente se puede tener acceso a la limpieza dental y algunas obturaciones provisionales, ya que las obturaciones definitivas son costosas y no hay buena situación económica para estos costos. De igual forma, las muelas pueden extraerse sin ningún inconveniente cuando las personas van de urgencias o con problemas periodontales. Sin embargo, la mayoría de los individuos manifiestan que hacen falta visitas domiciliarias periódicas por parte de los odontólogos en el puesto de salud, para que el conocimiento que instauren se mantenga a largo plazo (figura 1). 


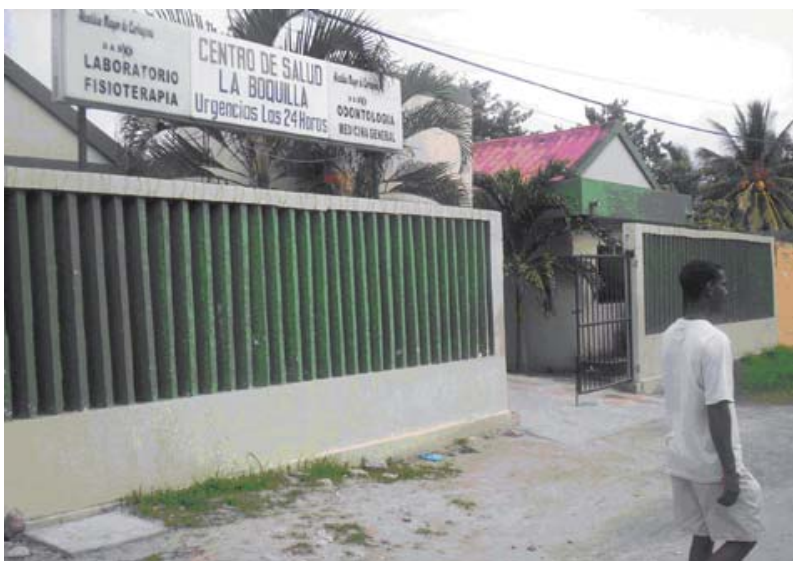

Figura 1. Puesto de Salud en la población Boquillera

\section{Origen y significado de los conocimientos en salud oral}

En primera instancia el origen de los discursos educativos en salud oral viene de diversos medios: televisión, radio, odontólogo, fundaciones en la población y algunos de las escuelas (figura 2). Con respecto a la primera fuente, ésta parece ser la principal, ya que es un medio visual que se ha convertido en el máximo entretenimiento $\mathrm{y}$, aunque en todos los hogares no existe, se reúnen permanentemente donde el familiar o vecino más cercano que lo tenga y de esta forma se puede estar al tanto de los programas que presentan. Los adultos manifiestan su gusto por novelas y noticieros y expresan que alguna publicidad contiene mensajes alusivos a la salud oral y han aprendido muchas estrategias preventivas, las cuales son reiteradas en expresiones como "Que los dientes se deben de lavar después de cada comida, se debe utilizar el enjuague bucal, hilo dental, esto lo he visto en la televisión". Por otra parte, también manifiestan que han aprendido la forma de lavarse los dientes con canciones de niños por televisión. En este mismo aspecto, el simbolismo que más ha influido en la

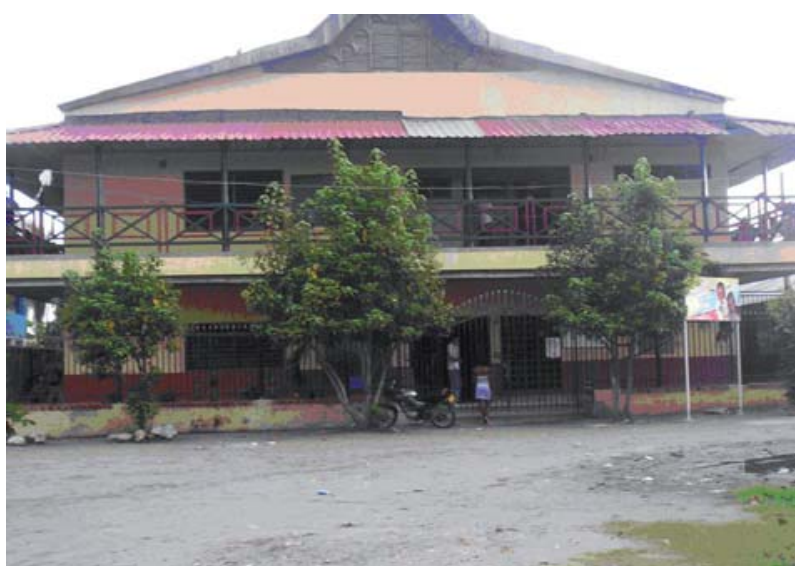

Figura 2. Fachada de la institución escolar del Corregimiento INETEB (Institución Técnica y Educativa de La Boquilla). conservación de estos mensajes ha sido la crema dental y el cepillo de dientes, los cuales dan sensación de limpieza y de buena salud.

Por otro lado, el odontólogo sigue siendo para ellos una figura importante para impartir el conocimiento de la prevención de enfermedades orales. No obstante, es claro que les hace falta que la enseñanza integre teoría y práctica alrededor del conocimiento social, ya que solamente con el aprendizaje impulsado desde el discurso cientificista se hace muy difícil adquirir las habilidades que se requieren para limpiarse los dientes.

\section{DISCUSIÓN}

La pertinencia de este trabajo investigativo esta relacionada con los innumerables beneficios que obtendrían los usuarios potenciales de los resultados. En primera instancia se podrían diseñar desde la Universidad de Cartagena programas de promoción de la salud y prevención de la enfermedad con estrategias educativas acordes con cada grupo social, brindándoles a las comunidades marginales más vulnerables la posibilidad de preservar sus órganos dentarios.

Estos elementos, que se encuentran representados en los resultados, han hecho que reflexionemos en torno a cuáles son los factores que hacen que se maneje una dualidad entre el conocimiento construido socialmente y este mismo puesto en práctica. Lo primero que salta a la luz de las explicaciones es la pobreza, debido a que ésta se ha convertido en un factor de influencia para tener buenos y eficaces elementos de higiene oral dentro del hogar. En esta comunidad los resultados difieren de este juicio de valor, ya que la mayoría de los habitantes cree que ser pobre no condiciona la enfermedad. Muchos tienen una vida lúgubre principalmente por la ausencia del dinero permanentemente y por los problemas que esto conlleva, lo que puede contribuir a la pérdida de las costumbres saludables y de un desarrollo humano visto desde la gestión y participación en procesos comunitarios tendentes a progresar como ser social y transformar su nivel de vida en la pirámide de la pobreza ${ }^{9}$.

En segunda instancia nos preguntamos por el elemento sociocultural, tratándose de una población sin muchas motivaciones de vanidad estética corporal e intelectual, debido a que los hombres son esclavos de su trabajo y su precaria situación económica los obliga a estar todo el tiempo buscando el diario y los escenarios laborales y pocos momentos de diversión no exigen una presencia estética, en donde la cavidad oral sea un componente importante. Por otro lado, las mujeres están dedicadas al hogar y cuidado de los niños en algunos casos y en otros a la cocina en restaurantes y negocios comerciales 
improvisados que son el sustento de la mayoría de las familias durante los fines de semana, los cuales no dejan espacio para eventos sociales que representen una importante presencia e imagen física de limpieza. Esto crea seres humanos tristes y sin un sentido de organización social, lo que contribuye a la pérdida de la identidad cultural ${ }^{10,11}$.

Por último, los niños conviven con un medio ambiente poco higiénico, insalubre, en donde, además de los juegos, no hay motivaciones adicionales hacia la limpieza y la buena presencia, lo que tampoco ha sido inculcado por los padres por el poco tiempo que les queda para monitorear su educación y sus buenas costumbres. Esto probablemente ha impulsado un estado de desidia social que logra interpretar parcialmente el por qué de sus ambigüedades en el tema de la salud oral (figura 3 ).

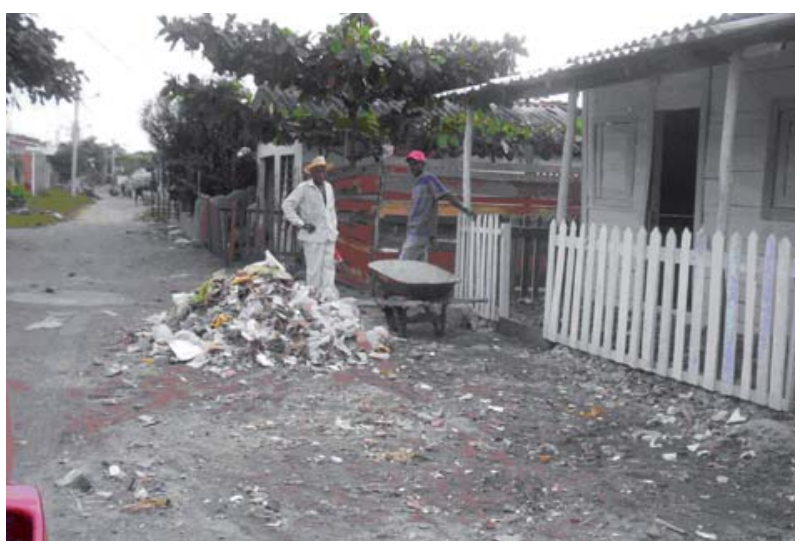

Figura 3. Proceso de recolección de basuras en el interior de la comunidad.

Desde esta perspectiva, todavía no se ha comprendido la importancia de las visitas periódicas a un centro de salud con el fin de hacerle seguimiento y prevenir las enfermedades orales. De igual forma, es claro que el odontólogo es el profesional mejor capacitado para esto, pero no se evidencian las diferencias entre la efectividad de este trabajo profesional y las acciones que se desarrollan desde el saber popular, ya que mientras éstas den resultado no es posible perder tiempo y dinero bajo las otras alternativas.

Otro aspecto importante para intentar comprender es la interpretación que la población tiene de la ingesta de crema dental durante el cepillado, especialmente en estas edades cuando los dientes permanentes se están formando, lo que puede ocasionar un aumento en la prevalencia de la fluorosis, la cual tiene unas características estéticas igual de importantes que la caries dental para el mantenimiento de la autoestima en niños escolares ${ }^{12}$.

Entre los estudios de representaciones sociales que se han realizado en el país en los últimos años se encuentran el de Nieva y Jácome ${ }^{13,14}$ en 1998, quienes evaluaron poblaciones urbanas marginales de ciudad Bolívar, en Bogotá. Los resultados dieron a conocer cómo la gente interpreta este proceso desde tres aspectos, como son lo estético, biológico y emocional. Por otro lado, en la ciudad de Cartagena realizamos un estudio cualitativo sobre representaciones sociales en $2008^{15}$ dirigido a padres de niños que son atendidos en las clínicas odontológicas de dos universidades de la región, encontrándose resultados diferentes en cuanto a la motivación hacia la higiene oral, siendo lo estético el principal aspecto para la asistencia al odontólogo. Por otra parte, en cuanto a percepciones de salud oral en condiciones como el embarazo, Fuentes et $\mathrm{al}^{16}$ en 2007 , en una población chilena, encontraron que el $70 \%$ de las mujeres que participaron en el estudio cree que el embarazo puede producir perdida de órganos dentarios y el $75 \%$ ha evidenciado sangrado de las encías, resultados muy similares a los encontrados en el presente estudio, lo que demuestra una vez más la influencia que ejercen las percepciones sobre la prevención de las enfermedades.

En este sentido y a la luz de estos resultados, se propone que la construcción de conocimientos para la adquisición y aplicación de buenos hábitos de higiene oral en la población de La Boquilla debe hacerse bajo una negociación de saberes, en donde el profesional tenga en cuenta el pensamiento y opinión del paciente como una alternativa más de salud, debido a que en la actualidad existen saberes validados socialmente con características de verdad absoluta, al igual que conocimientos validados científicamente, sin embargo, es claro que la mejor forma de llegar a conocer la realidad es a través de la complementación de saberes. Hoy en día es tan falible el conocimiento ideológico como el científico, representado esto por los innumerables cambios que se presentan en la rueda de la ciencia, los cuales van corrigiendo los conocimientos que durante muchos años se consideraban como válidos y se imponen los que actualmente resisten la crítica de la comunidad científica actual, más por incapacidad de encontrar los errores por parte de los académicos que por lo que representan ${ }^{17}$.

La comprensión de esta realidad social debe permitir que el profesional de hoy asuma una actitud sin dogmas, enmarcando su comportamiento a partir del reconocimiento del otro como un ser con igual capacidad para utilizar herramientas preventivas que le permitan resolver los problemas relacionados con las enfermedades orales, sin necesidad de haber tenido acceso a una universidad ${ }^{18}$.

Por otro lado, se espera que este aporte sea significativo para las instituciones de salud del distrito de Cartagena - Colombia, las cuales pueden utilizar este mismo modelo en poblaciones con comporta- 
mientos socioculturales diferentes, cuyos resultados serían únicos y utilizados sólo en estos individuos.

Teniendo en cuenta el análisis realizado durante todo el proceso, se concluye que las representaciones sociales sobre la salud y la enfermedad oral en la población de La Boquilla ejercen una influencia en la apropiación del conocimiento dentro de su núcleo familiar, hasta el punto de lograr confrontarlo con el conocimiento científico que transmiten los profesionales de la salud y de esta forma sacar conclusiones individuales basadas en una reflexión desde una perspectiva empírica e ideológica. Además, se evidenciaron interpretaciones que estaban influenciadas por la cultura, el trabajo, la pobreza, las emociones etc., elementos éstos poco racionales desde lo científico, los cuales logran reconocer a un ser social que puede tener comportamientos y actitudes saludables con explicaciones diferentes a la cultura occidental, pero tratando de buscarle una solución al problema desde diferentes dimensiones.

\section{AGRADECIMIENTOS}

A la institución educativa INETEB y a la comunidad de La Boquilla - Cartagena, por la gestión y los aportes durante el proceso de recolección de la información. A la Universidad de Cartagena por el apoyo administrativo para el desarrollo de esta investigación.

\section{BIBLIOGRAFÍA}

1. González F, Alfaro L, Nieto C, Carmona L. Evaluación de las condiciones de salud oral y los conocimientos en niños escolarizados entre 5 y 14 en la población de La Boquilla, Cartagena de Indias 2004. Rev Científica Odontología Universidad El Bosque. 2006; 12 (1):25-34.

2. Ministerio de Salud - República de Colombia, Centro Nacional de Consultoría CNS: III- ENSAB III; Tomo VII. Bogotá: Lito Servicios ALER; 1999.

3. Franco Á, Martínez C, González M. Los programas preventivos de salud bucal en el contexto del Sistema de Seguridad Social en Salud. Medellín: Copi@net, comunicación integral; 2003. p. 13-15.

4. Briones G. Evaluación de programas sociales. México: TriIlas; 1998.
5. Sistema General de Seguridad Social Integral. Ley 100 de 1993. República de Colombia. Bogotá: Ministerio de Salud; 1995.

6. Barnes, B. "El Problema del Conocimiento", en León Olivé (ed.), La Explicación Social del Conocimiento. México: Dirección General de Publicaciones de la Universidad Nacional Autónoma de México; 1985.

7. Sandoval C. Investigación cualitativa, Módulos Icfes, Especialización Métodos y técnicas de Investigación social. Bogotá; 2003.

8. González F, Oyola E, Cadrazco F, Herrera Y. Representaciones sobre los escenarios de prácticas en salud oral de los habitantes de La Boquilla - Cartagena de Indias. Rev. CES Odontología. 2007; 20(1):9-19.

9. González F, Carmona L, Agudelo Y, Campo P. Análisis social de las familias del barrio Nelson Mándela de Cartagena de Indias, en cuanto a sus conocimientos en salud y enfermedad general y oral. Rev. Usta Salud Odontología. 2006; 5(2):115-21.

10. Mariño G. El dialogo cultural. Reflexiones en torno a su fundamentación, su metodología y su didáctica. Bogotá: Dimensión educativa; 1994

11. Colombo E. El imaginario social. Montevideo: Altamira; 1993.

12. Franco A, Martignón E, Saldarriaga A, González M, Luna L, Ocampo A. et al. Total fluoride intake in children aged 22-35 months in four Colombian cities. Community Dent Oral Epidemiol. 2005; 33:1-8.

13. Nieva B, Jácome S. Representaciones sociales del proceso Salud Enfermedad Oral en Poblaciones Urbano Marginales y su relación con los discursos y las prácticas institucionales. Primera parte. Rev. Federación Odontológica Colombiana. 1999; 57:30-32.

14. Nieva B, Jácome S. Representaciones sociales del proceso Salud Enfermedad Oral en Poblaciones Urbano Marginales y su relación con los discursos y las prácticas institucionales. Segunda parte. Rev. Federación Odontológica Colombiana. 1999; 58:10-12.

15. González F, Redondo A, Díaz K. Representaciones sociales sobre la higiene oral de los niños menores de cinco años que asisten a la clínica Cartagena de Indias Universidad del Sinú y Universidad de Cartagena. Rev. Duazary. 2009; 6(1):38-46.

16. Fuentes R, Oporto G, Alarcón AM, Bustos L, Prieto R, Rico $H$. Opiniones y creencias de embarazadas en control prenatal relacionadas con salud oral y embarazo. Av Odontoestomatol. 2009; 25(3):147-54.

17. Balbín J. El diálogo de saberes una búsqueda en: Del mesianismo y el populismo al dialogo de saberes. Aportes 33 . México: Dimensión Educativa; 1990.

18. González F, Carmona L, Campo P. Prevención de la enfermedad oral en familias desplazadas por la violencia de la comunidad de Nelson Mándela Cartagena - Colombia. Rev CES Odontología. 2008; 21(1):17-26. 